\title{
MONTE CARLO DEPLETION UNDER LEAKAGE- CORRECTED CRITICAL SPECTRUM VIA ALBEDO SEARCH
}

\author{
SUNGHWAN YUN and NAM ZIN CHO* \\ Korea Advanced Institute of Science and Technology \\ Daejeon, 305-701, Korea \\ *Corresponding author. E-mail : nzcho@kaist.ac.kr \\ Received November 24, 2009 \\ Accepted for Publication May 4, 2010
}

While the deterministic lattice physics/depletion codes use leakage-corrected critical spectrum (although approximate due to the $\mathrm{B}_{1}$ buckling search employed), Monte Carlo depletion codes currently in use do not have such a feature in spite of their heterogeneity and continuous-energy modeling capability. This paper describes an approach to Monte Carlo depletion with leakage-corrected critical spectrum derived from first principles. This is based on the concept of albedo eigenvalue treated as weight of the reflected neutron in Monte Carlo simulation.

KEYWORDS : Monte Carlo Depletion, Critical Spectrum, Albedo, Leakage Correction, MONTEBURNS

\section{INTRODUCTION}

In conventional deterministic lattice physics/depletion codes, e.g., CASMO [1] or HELIOS [2], a leakage-corrected critical spectrum is used for the depletion of burnable nuclides and the buildup of fission products. The critical spectrum is obtained by $\mathrm{B}_{1}$ (linearly anisotropic scattering) buckling search based on multigroup, homogenized medium asymptotic spectrum (under energy-space separability assumption). However, the deterministic lattice physics codes have limitations in treating complex geometry and continuous-energy.

The Monte Carlo method overcomes these limitations, but the Monte Carlo depletion codes currently in use such as the MONTEBURNS code [3] do not use the leakagecorrected critical spectrum [4]. Instead they use no-leakage critical spectrum.

In our previous studies [5-7], we introduced an approach to Monte Carlo depletion with leakage-corrected critical spectrum, for the first time to our knowledge, in which the streaming (leakage) term of the transport equation is treated as an eigenvalue term and transformed into a specialized albedo boundary condition problem. Thus, the approach is neutron transport simulation based on first principles (without relying on ad-hoc assumptions such as the $\mathrm{B}_{1}$ buckling search). This approach was implemented in the MONTEBURNS code. In our new approach, the MCNP5 engine [8] was modified to solve the albedo boundary condition problem, while the MONTEBURNS code was modified to obtain an appropriate albedo value at current burnup time step by the secant method.
The leakage-corrected critical spectrum found in the Monte Carlo simulation can be used in depleting the nuclides and in generating multigroup cross sections of the fuel assemblies to be used in the whole-core analysis, thus replacing the deterministic lattice physics calculation by the Monte Carlo calculation. In this paper, the method of Monte Carlo depletion with leakage-corrected critical spectrum is described in detail and the implementation is refined. Also reported are several numerical tests performed on representative $\mathrm{UO}_{2}$ and MOX fuel assemblies.

\section{LEAKAGE CORRECTIONS USING SPECIALIZED ALBEDO BOUNDARY CONDITION}

Consider the following steady-state transport equation to be simulated by a continuous-energy Monte Carlo code:

$$
\begin{aligned}
& \vec{\Omega} \cdot \nabla \psi(\vec{r}, \vec{\Omega}, E)+\sigma_{t}(\vec{r}, E) \psi(\vec{r}, \vec{\Omega}, E) \\
& =\int d \vec{\Omega}^{\prime} \int d E^{\prime} \sigma_{s}\left(\vec{r}, E^{\prime} \rightarrow E, \vec{\Omega}^{\prime} \cdot \vec{\Omega}\right) \psi\left(\vec{r}, \vec{\Omega}^{\prime}, E^{\prime}\right) \\
& +\frac{\chi(E)}{k_{\text {eff }}} \int d \vec{\Omega}^{\prime} \int d E^{\prime} v \sigma_{f}\left(\vec{r}, E^{\prime}\right) \psi\left(\vec{r}, \vec{\Omega}^{\prime}, E^{\prime}\right),
\end{aligned}
$$

where $\psi$ is neutron angular flux, and other notations are standard [9].

First, define a specialized albedo,

$$
\alpha \equiv \frac{\psi\left(\vec{r}, \vec{\Omega}^{\prime}, E\right)}{\psi(\vec{r}, \vec{\Omega}, E)}, \text { for } \vec{n} \cdot \vec{\Omega}^{\prime}=-\vec{n} \cdot \vec{\Omega}<0, \vec{r} \in \Gamma \text {. }
$$


Now, if Eq. (1) is integrated over angular and spatial variables in a volume, the first term (leakage term) becomes

$$
\begin{aligned}
\int_{V} \int_{4 \pi} \nabla \cdot \vec{\Omega} \psi(\vec{r}, \vec{\Omega}, E) d \vec{\Omega} d V & =\int_{\Gamma} \vec{n} \cdot \int_{4 \pi} d \vec{\Omega} \vec{\Omega} \psi(\vec{r}, \vec{\Omega}, E) d \Gamma \\
& =\int_{\Gamma}\left[J^{+}(\vec{r}, E)-J^{-}(\vec{r}, E)\right] d \Gamma \\
& =(1-\alpha) \int_{\Gamma} J^{+}(\vec{r}, E) d \Gamma
\end{aligned}
$$

Thus, we note that a $k_{\text {eff }}$-eigenvalue problem can be changed to a (1- $\alpha)$-eigenvalue problem for an assembly to be depleted. Eq. (3) also indicates that $\alpha$ should be unity in

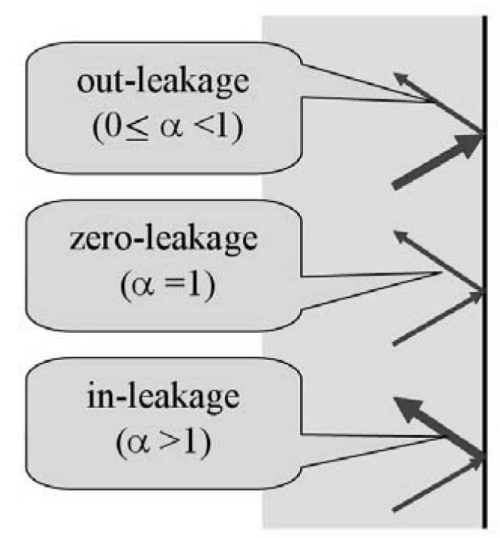

Fig. 1. Monte Carlo Reflects a Neutron with Weight $\alpha$ at a Surface critical $\left(k_{\infty}=1\right)$ lattice problem, while $\alpha<1$ in supercritical $\left(k_{\infty}>1\right)$ lattice problem to achieve criticality $\left(k_{e f f}=1\right)$ and vice versa. By varying albedo $\alpha$, we can make in-leakage or out-leakage and obtain critical $\left(k_{\text {eff }}=1\right)$ spectrum. This is depicted in Fig. 1.

In our approach, the MCNP5 code is wrapped around by the secant method. Thus, the MCNP5 code is modified to solve the following albedo boundary balance equation for eigenvalue $k_{\text {effi, }}$, with given albedo $\alpha_{i}$ by adjusting the reflected Monte Carlo particle weight by $\alpha_{i}$ :

$$
\begin{gathered}
\vec{\Omega} \cdot \nabla \psi(\vec{r}, \vec{\Omega}, E)+\sigma_{t}(\vec{r}, E) \psi(\vec{r}, \vec{\Omega}, E) \\
=\int d \overrightarrow{\Omega^{\prime}} \int d E^{\prime} \sigma_{s}\left(\vec{r}, E^{\prime} \rightarrow E, \vec{\Omega}^{\prime} \cdot \vec{\Omega}\right) \psi\left(\vec{r}, \vec{\Omega}^{\prime}, E^{\prime}\right) \\
+\frac{\chi(E)}{k_{e f f, i}} \int d \overrightarrow{\Omega^{\prime}} \int d E^{\prime} v \sigma_{f}\left(\vec{r}, E^{\prime}\right) \psi\left(\vec{r}, \vec{\Omega}^{\prime}, E^{\prime}\right), \\
\psi\left(\vec{r}, \vec{\Omega}^{\prime}, E\right)=\alpha_{i} \psi(\vec{r}, \vec{\Omega}, E), \text { for } \vec{n} \cdot \vec{\Omega}^{\prime}=-\vec{n} \cdot \vec{\Omega}<0, \vec{r} \in \Gamma,
\end{gathered}
$$

where $i$ is the iteration index of the secant method. Then, $\alpha$ is updated until $k_{\text {eff }}$ becomes 1.0 as follows:

$$
\alpha_{i+1}=\alpha_{i}-\frac{k_{e f f, i}\left(\alpha_{i-1}-\alpha_{i}\right)}{k_{e f f, i-1}-k_{e f f, i}},
$$

where $\alpha_{1}=1.0$ and $\alpha_{2}=0.5$ are used for the initial values. $3 \sigma$ (the standard deviation of $k_{\text {eff } i}$ ) is chosen as an iteration

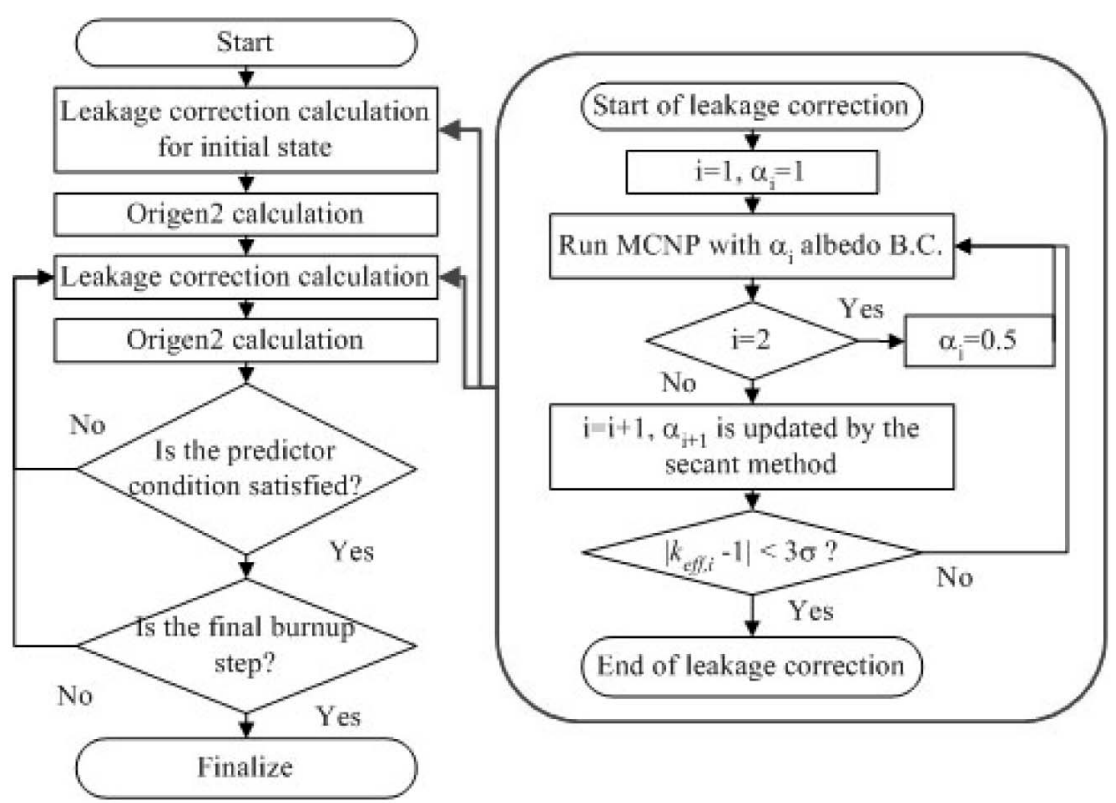

Fig. 2. Flow Chart of Leakage-corrected Critical Spectrum Depletion in the MONTEBURNS-Lk Code 
stop criterion. A flow chart of the leakage correction in the modified MONTEBURNS code (named MONTEBURNSLk) is shown in Fig. 2.

The albedo considered in Eq. (2) can be generalized as

$$
\begin{gathered}
\psi\left(\vec{r}, \vec{\Omega}^{\prime}, E\right) \equiv \alpha\left(\vec{r}, \vec{\Omega}^{\prime}, E\right) \psi(\vec{r}, \vec{\Omega}, E), \\
\text { for } \vec{n} \cdot \vec{\Omega}^{\prime}=-\vec{n} \cdot \vec{\Omega}<0, \vec{r} \in \Gamma,
\end{gathered}
$$

and if information of the neighbors (e.g., another assembly or reflector) is available as in local (assembly)/global (whole-core) iterative calculation, the generalized albedo $\alpha$ could be provided from the core-level calculation (albeit approximately in discrete form). An application of this procedure is presented in Ref. [10].

\section{NUMERICAL RESULTS}

\section{1 $\mathrm{UO}_{2}$ Fuel Assembly Problem}

A $\mathrm{UO}_{2}$ assembly problem composed of $18 \times 18$ fuel rods is considered as the first test problem. A detailed description of the first test problem is shown in Fig. 3 and

Table 1. Material Properties of the $\mathrm{UO}_{2}$ Fuel Assembly Problem

\begin{tabular}{c|c|c}
\hline Region & Material & Radius $[\mathrm{cm}]$ \\
\hline Fuel & 4 w/o UO $\left(\rho=10.89\left[\mathrm{~g} / \mathrm{cm}^{3}\right]\right)$ & 0.4095 \\
\hline Gap & Water $\left(\rho=1.0\left[\mathrm{~g} / \mathrm{cm}^{3}\right]\right)$ & 0.4180 \\
\hline Clad & Zircalory-4 $\left(\rho=6.489\left[\mathrm{~g} / \mathrm{cm}^{3}\right]\right)$ & 0.4750 \\
\hline Moderator & Water $\left(\rho=1.0\left[\mathrm{~g} / \mathrm{cm}^{3}\right]\right)$ & - \\
\hline \multicolumn{3}{c}{}
\end{tabular}
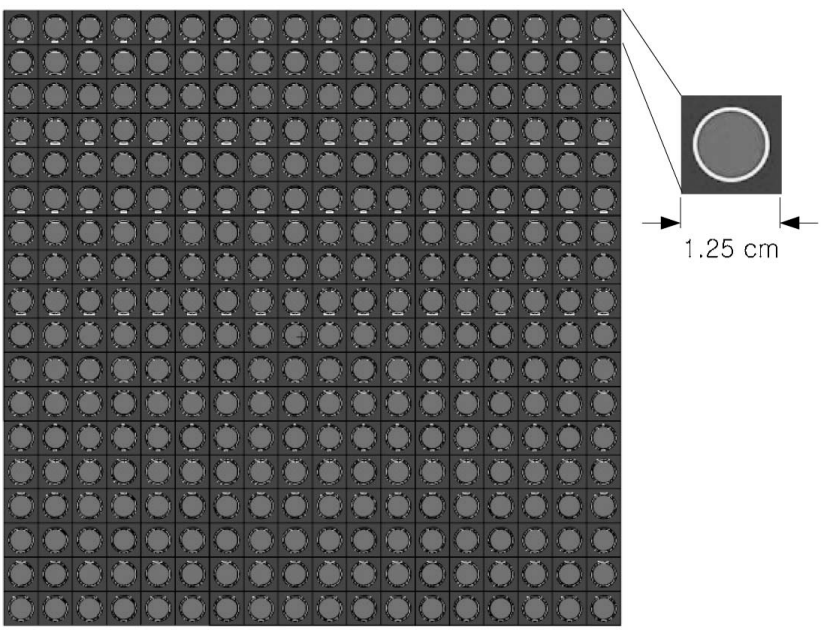

Fig. 3. Configuration of the $\mathrm{UO}_{2}$ Fuel Assembly Problem
Table 1. For the MONTEBURNS-Lk calculation, 217 isotopes in ENDF/B-VI.6 continuous-energy cross section libraries are used with 10,000 histories per generation and 50/100 inactive/active generations, while 20 outer/ 100 inner burnup steps are used. For this test problem, the assembly is used as one tally region in the depletion calculation, thus assembly-averaged reactor physics parameters are evaluated.

A comparison of the $k_{\infty}$ along burnup and the albedo values is shown in Fig. 4, while normalized flux spectra are shown in Fig. 5. As described in the previous section, the estimated albedo $\alpha$ becomes unity at $\sim 39$ [GWD/MTU]

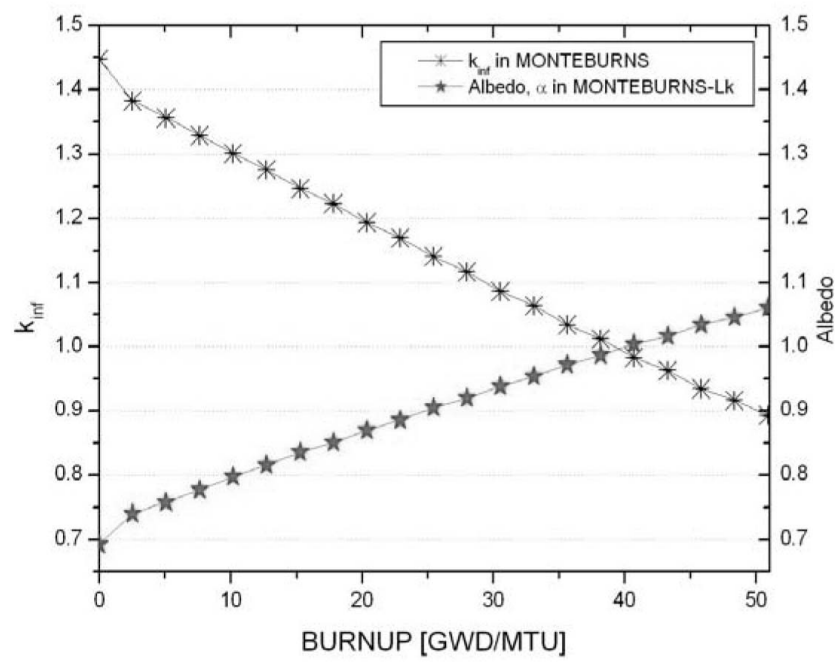

Fig. 4. Trend of $k_{\infty}$ and Albedo vs. Burnup in the $\mathrm{UO}_{2}$ Fuel Assembly

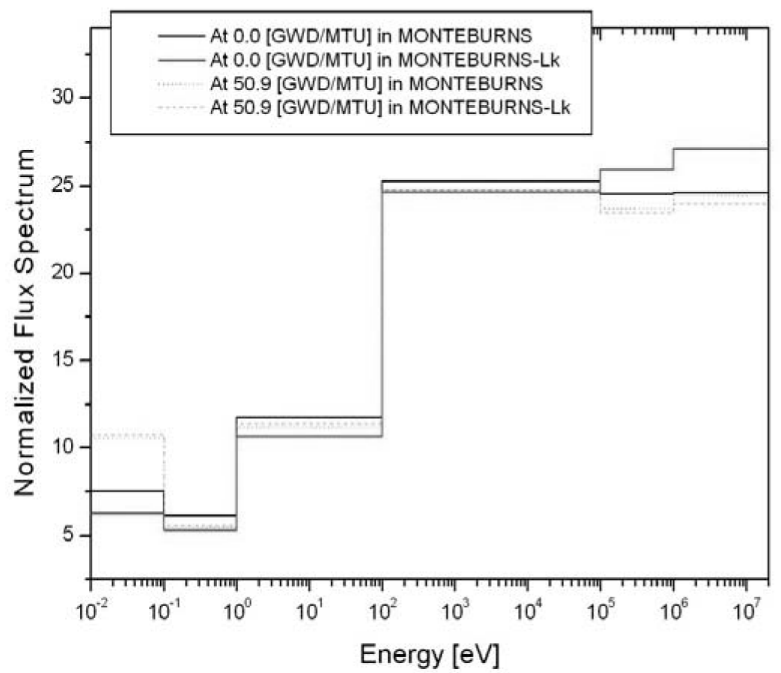

Fig. 5. Normalized Neutron Flux Spectra of Two Burnup Stages in the $\mathrm{UO}_{2}$ Fuel Assembly 


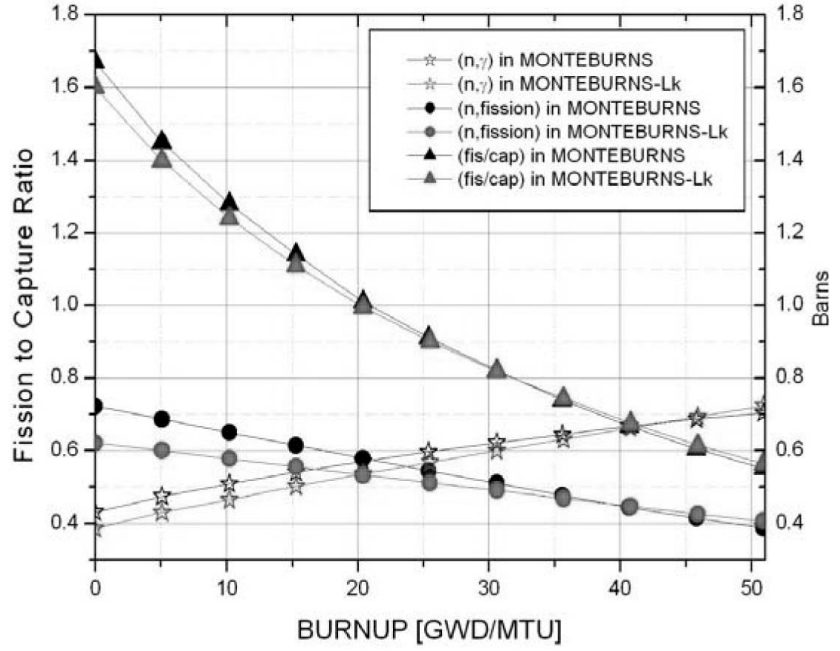

Fig. 6. Fission to Capture Ratio and Resulting Equivalent OneGroup Cross Sections vs. Burnup in the $\mathrm{UO}_{2}$ Fuel Assembly

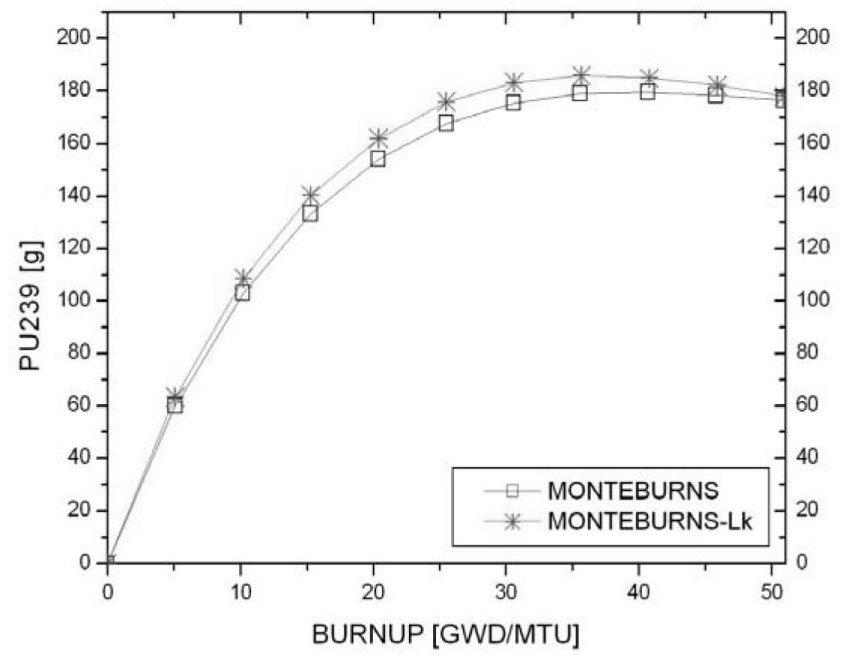

Fig. 7. Comparison of $\mathrm{Pu}^{239}$ vs. Burnup in the $\mathrm{UO}_{2}$ Fuel Assembly

burnup (at which point, $k_{\infty}=k_{\text {eff }}=1$ ). The leakage-corrected spectra are harder than the conventional case at initial state (when $\alpha<1$ ), while the spectra in the two cases tend to be closer but show inverse tendency at 50.9 [GWD/MTU] burnup (when $\alpha>1$ ).

Due to the difference in spectrum, the fission to capture ratio and the resulting equivalent one-group cross sections of fuel material in the leakage corrected case show discrepancies as in Fig. 6. The variations in the resulting critical spectra and equivalent one-group fission cross sections show the same tendencies with those of the deterministic CASMO-3 calculations with/without critical buckling search reported in Ref. [4]. The discrepancies in depleted or produced nuclides, e.g., $\mathrm{Pu}^{239}$, are also shown in Fig. 7.

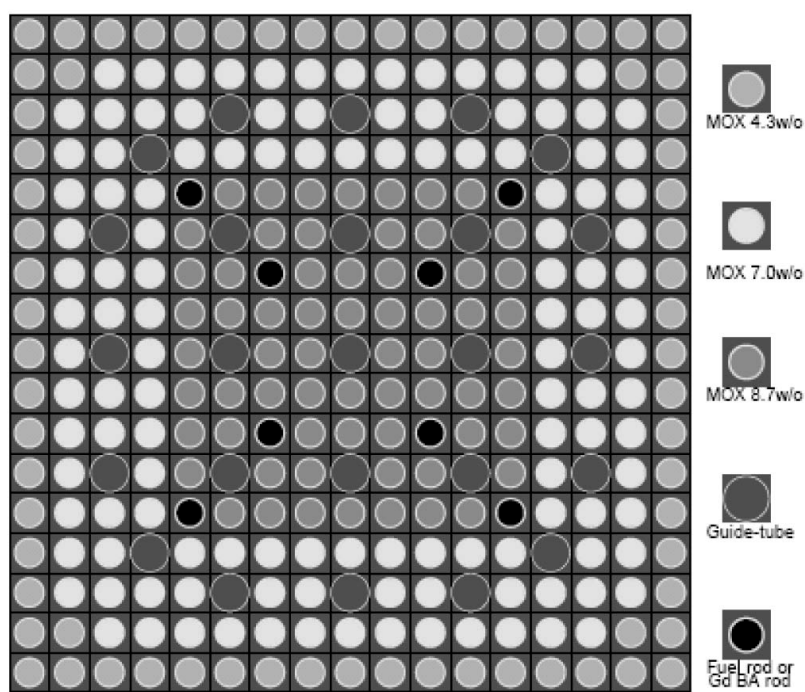

Fig. 8. Configurations of MOX Fuel Assembly

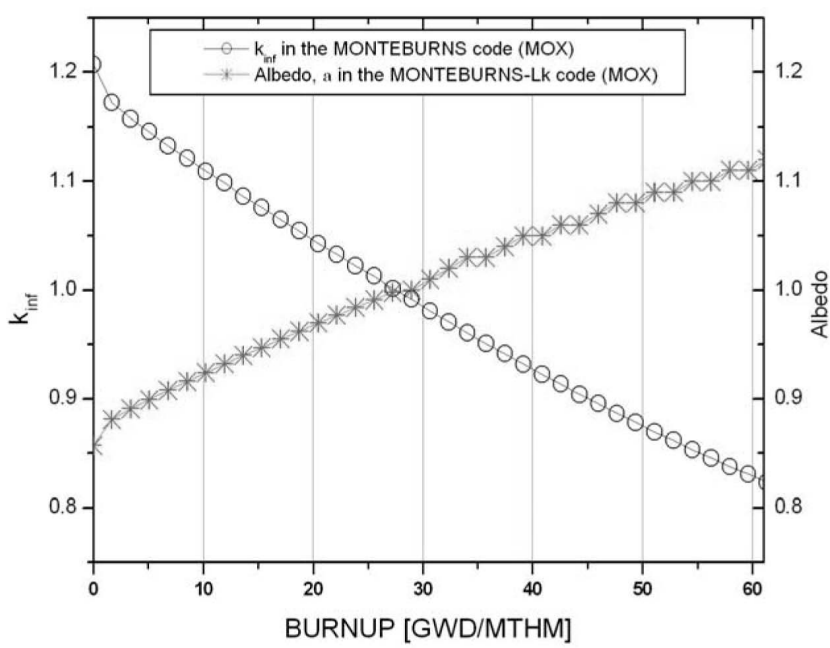

Fig. 9. Trend of $k_{\infty}$ and Albedo vs. Burnup in MOX Fuel Assembly

\subsection{MOX Fuel Assembly Problem}

The second test problem is a MOX fuel assembly, which is shown in Fig. 8, and the detail specifications are given in Ref. [11]. For the MONTEBURNS-Lk calculation, 217 isotopes in ENDF/B-VI.6 continuous-energy cross section libraries are used with 10,000 histories/generation and 100/300 inactive/active generations, while 20 outer/ 100 inner burnup steps are used. For z-direction, $20 \mathrm{~cm}$ height is used with reflective B.C. For this second test problem, each pin region is used as a tally region in the depletion calculation, thus pin power distributions are evaluated.

Comparisons of $k_{\infty}$ and albedo values along burnup in MOX assembly are shown in Fig. 9. 


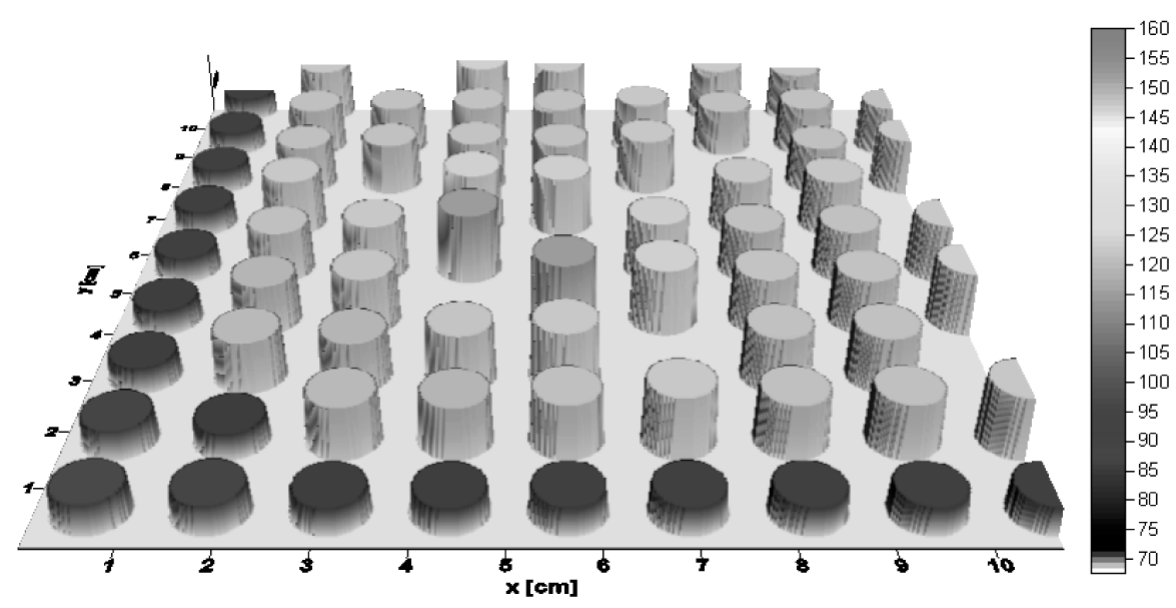

Fig. 10. Pin Power Density Distribution $\left[\mathrm{W} / \mathrm{cm}^{3}\right]$ at BOC in a Quarter Assembly of MOX Fuel Using the Conventional MONTEBURNS Code

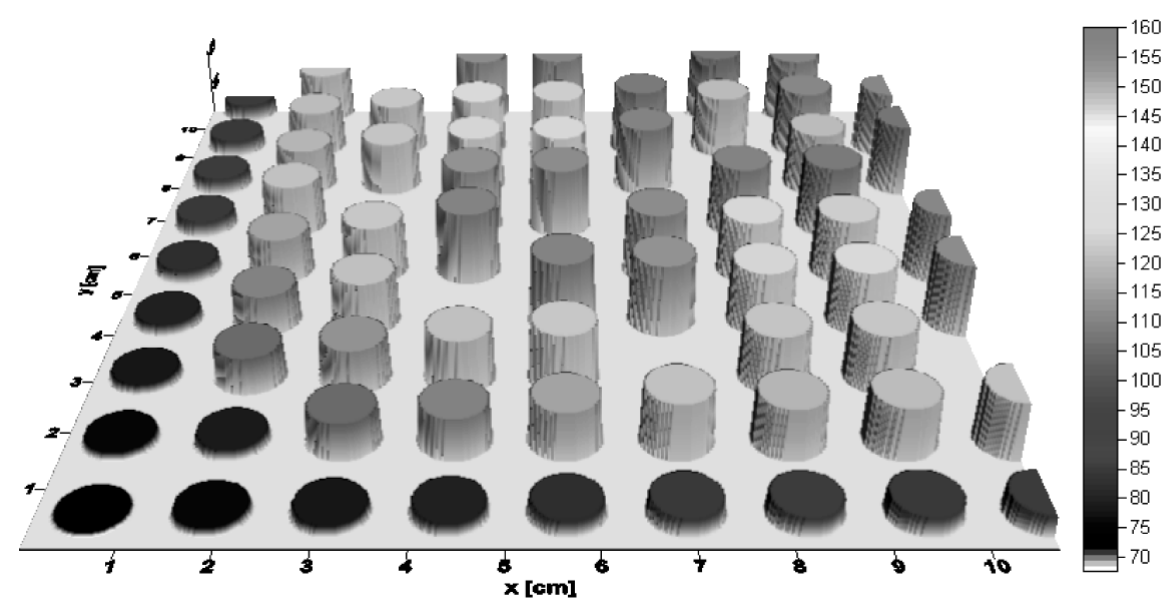

Fig. 11. Pin Power Density Distribution $\left[\mathrm{W} / \mathrm{cm}^{3}\right]$ at BOC in a Quarter Assembly of MOX Fuel Using the MONTEBURNS-Lk Code

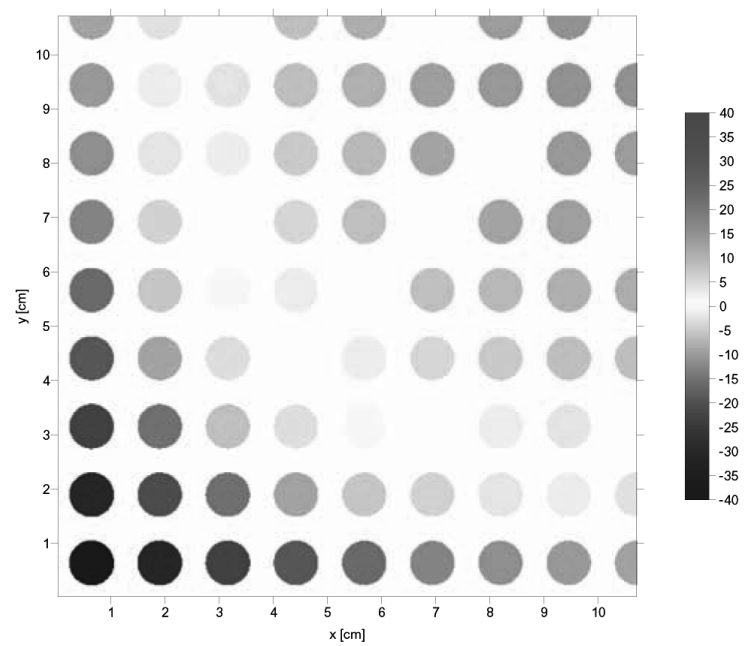

Fig. 12. Relative Difference [\%] in Pin Power Density Distribution at BOC in a Quarter Assembly of MOX Fuel
The pin power density distributions and their relative differences at BOC are shown in Figures 10, 11, and 12. The maximum pin power density in the conventional MONTEBURNS calculation is $149.8\left[\mathrm{~W} / \mathrm{cm}^{3}\right]$, while it is $156.8\left[\mathrm{~W} / \mathrm{cm}^{3}\right]$ in the MONTEBURNS-Lk calculation. The MONTEBURNS-Lk calculation shows $-40.67 \%$ in minimum and $14.29 \%$ in maximum difference.

The pin power density distributions and their relative differences at EOC (61.33 [GWD/MTHM]) are shown in Figures 13, 14, and 15. The maximum pin power density in the conventional MONTEBURNS calculation is $137.20\left[\mathrm{~W} / \mathrm{cm}^{3}\right]$, while it is $137.80\left[\mathrm{~W} / \mathrm{cm}^{3}\right]$ in the MONTEBURNS-Lk calculation. The MONTEBURNSLk calculation shows $-15.33 \%$ in minimum and $23.09 \%$ in maximum difference. The differences in pin power distributions are smaller than those at BOC, while they show opposite tendency.

The relative differences in $\mathrm{Pu}^{239}$ pin inventories at 


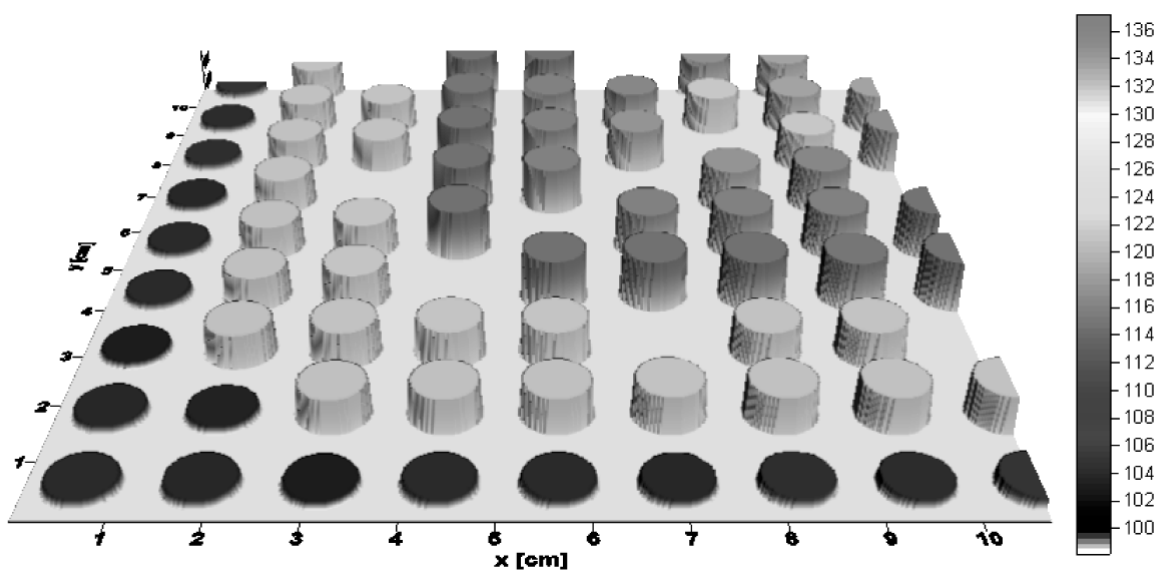

Fig. 13. Pin Power Density Distribution $\left[\mathrm{W} / \mathrm{cm}^{3}\right]$ at EOC in a Quarter Assembly of MOX Fuel Using the Conventional MONTEBURNS Code

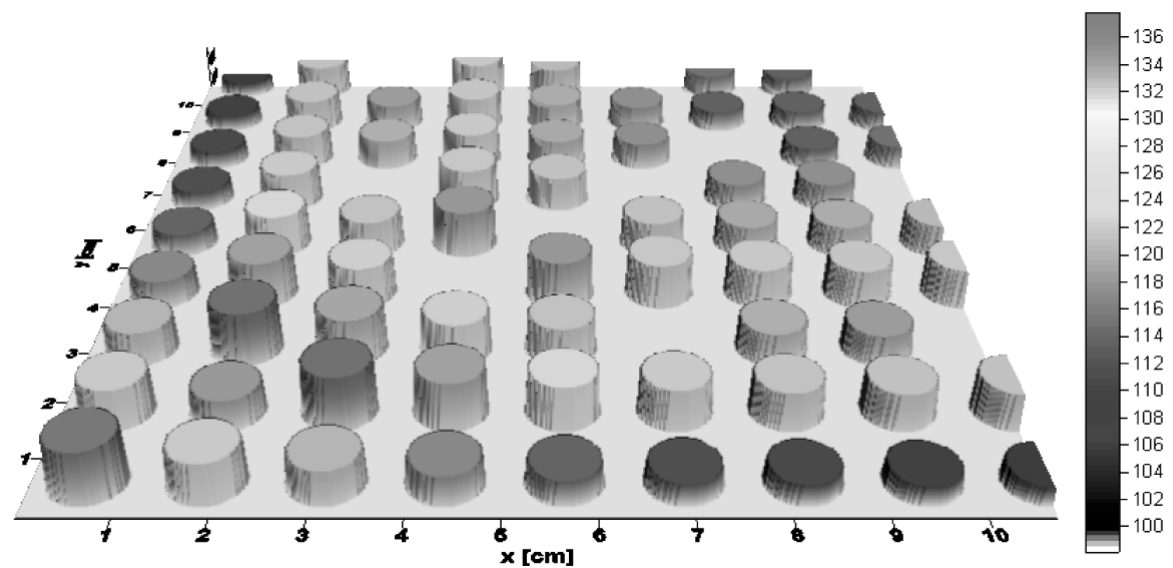

Fig. 14. Pin Power Density Distribution $\left[\mathrm{W} / \mathrm{cm}^{3}\right]$ at EOC in a Quarter Assembly of MOX Fuel Using the MONTEBURNS-Lk Code

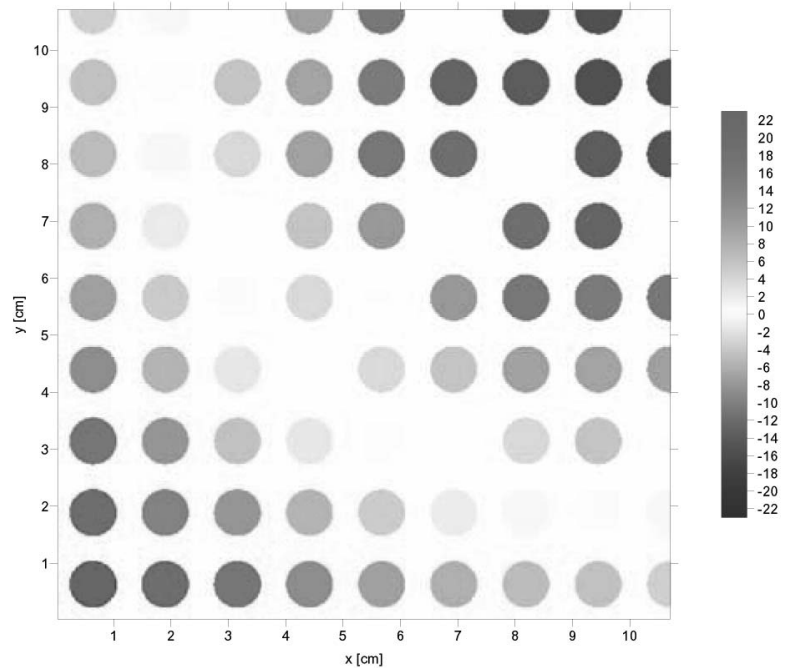

Fig. 15. Relative Difference [\%] in Pin Power Density Distribution at EOC in a Quarter Assembly of MOX Fuel

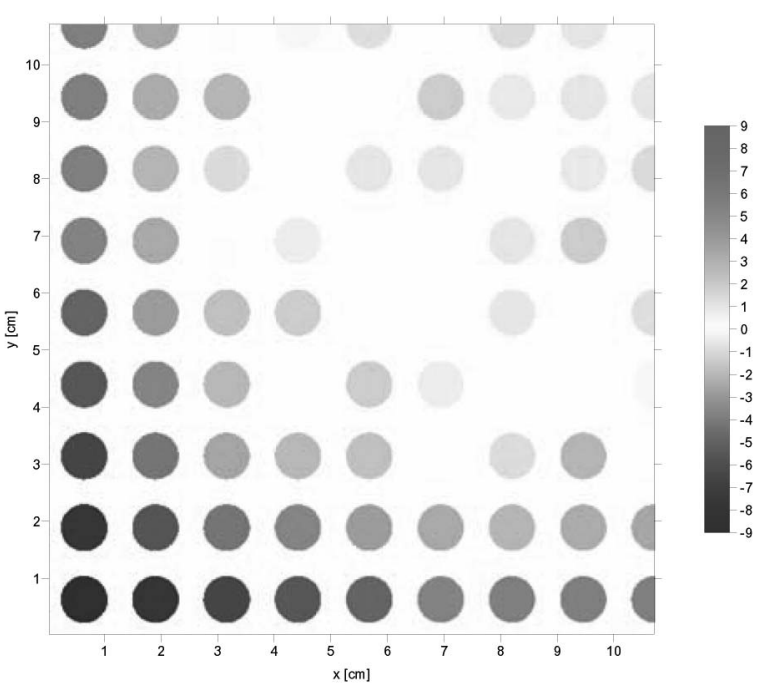

Fig. 16. Relative Difference [\%] in $\mathrm{Pu}^{239}$ Pin Inventories at EOC in a Quarter Assembly of MOX Fuel 


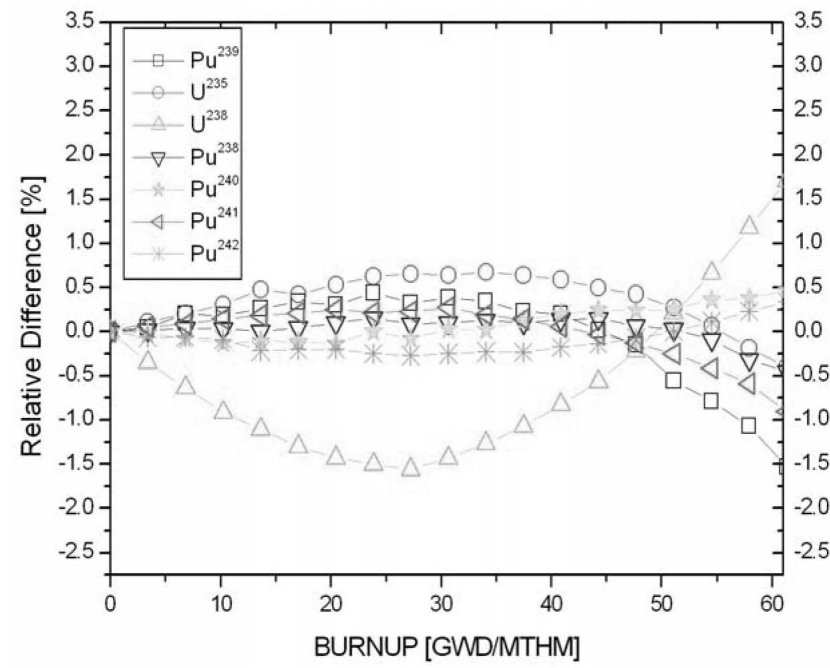

Fig. 17. Relative Difference [\%] in Plutonium Isotopes, $\mathrm{U}^{238}$, and $\mathrm{U}^{235}$ Inventories in a Quarter Assembly of MOX Fuel

EOC are shown in Fig. 16, while those in plutonium isotopes, $\mathrm{U}^{238}$, and $\mathrm{U}^{235}$ inventories in a quarter assembly of MOX fuel along burnup are shown in Fig. 17. The leakage-corrected critical spectra affect not only the spatial distributions of heavy isotope inventories, but also the burnup dependent variations of heavy isotope inventories.

\section{CONCLUSIONS}

A Monte Carlo depletion method with leakagecorrected critical spectrum was described, based on the concept of albedo eigenvalue treated as weight of the reflected neutron. The method was implemented in the MONTEBURNS code that calls MCNP5 using the albedo as boundary condition. Our new Monte Carlo depletion method provides rearranged critical neutron flux distribution in both energy spectra and spatial distributions (energy and space are coupled), which leads to differing depletion characteristics compared to that of the conventional Monte Carlo depletion. Appendix provides a simple description how the spectra are changed by the albedo search implemented in the Monte Carlo simulation.

Numerical results on $\mathrm{UO}_{2}$ and MOX fuel assemblies using our new method were also presented to compare the depletion characteristics between the leakagecorrected case and the conventional case. The pin power density distribution showed significant difference at BOC $(-40.67[\%] \sim 14.29[\%])$, while it showed less difference at EOC $(-15.33[\%] \sim 23.09[\%])$. The $\mathrm{Pu}^{239}$ inventory in the assembly showed $1.71[\%]$ relative difference at EOC, while $\mathrm{Pu}^{238}, \mathrm{Pu}^{240}, \mathrm{Pu}^{241}$ and $\mathrm{Pu}^{242}$ showed $-0.45[\%], 0.47[\%],-0.91[\%]$ and $0.34[\%]$ differences at EOC, respectively.

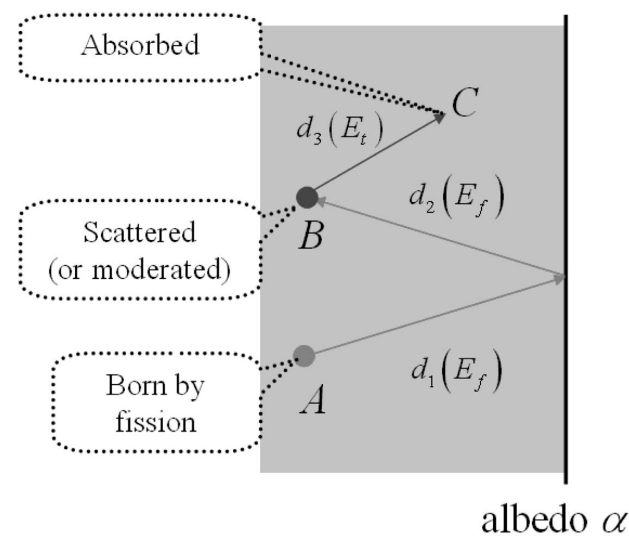

Fig. A1. Monte Carlo Particle Flights in a Region with Albedo $\alpha$ Surface

\section{APPENDIX}

The leakage-corrected critical spectrum of a supercritical $\left(k_{\infty}>1\right)$ lattice problem should be harder than the critical spectrum with no leakage, in order to decrease the fission reaction ratio (or the fission to capture ratio), while the leakage-corrected critical spectrum of a subcritical $\left(k_{\infty}<1\right)$ lattice problem should be softer than the critical spectrum with no leakage, in order to increase the fission reaction ratio (or the fission to capture ratio). Using the specialized albedo boundary condition, proposed in this paper, this phenomenon is induced in the Monte Carlo procedure.

For illustration, in Fig. A1, a simple homogeneous system is considered with a constant albedo boundary condition defined as $\alpha$. A Monte Carlo particle (or neutron) is born by fission at position $A$ with weight $w$ and high energy $E_{f}$, and flies to the boundary surface (distance $d_{1}$ ) and reflects to position $B$ (distance $d_{2}$ ) with changed weight $\alpha w$. Suppose that the neutron is then scattered (or moderated to low $E_{t}$ ) and absorbed at position $C$ (distance $d_{3}$ ). Then, the volume-integrated track-length tally fluxes are estimated as

$$
\begin{gathered}
\phi_{f}=w d_{1}+w \alpha d_{2}, \\
\phi_{t}=w \alpha d_{3} .
\end{gathered}
$$

Then, the ratios of $\phi_{f}$ to $\phi_{t}$ for various $\alpha$ are easily derived as follows:

$$
\frac{\phi_{f}}{\phi_{t}}=\frac{w d_{1}+w \alpha d_{2}}{w \alpha d_{3}}=\frac{d_{1}+\alpha d_{2}}{\alpha d_{3}}\left\{\begin{array}{l}
>\frac{d_{1}+d_{2}}{d_{3}}, \text { for } \alpha<1, \\
=\frac{d_{1}+d_{2}}{d_{3}}, \text { for } \alpha=1, \\
<\frac{d_{1}+d_{2}}{d_{3}}, \text { for } \alpha>1 .
\end{array}\right.
$$


Eq. (A3) indicates that, if $\alpha<1$, the spectrum is hardened and vice versa. This is borne out in the results of Fig. 5 in Section 3.1.

\section{REFERENCES}

[ 1 ] M. Edenius and B. H. Forssen, "CASMO-3 A Fuel Assembly Burnup Program User's Manual,” Studsvik/NFA-89-3, Rev. 2, Studsvik AB, 1992.

[2] HELIOS Methods, Studsvik ${ }^{\mathrm{TM}}$ Scandpower, 01 April, 2000.

[ 3 ] D. L. Poston and H. R. Trellue, "User's Manual, Version 2.0 for MONTEBURNS Version 1.0," LA-UR-99-4999, Los Alamos National Laboratory, 1999.

[4] J. C. Davis and J. C. Lee, "Comparison of Monte Carlo and Deterministic Depletion Codes for LWR Fuel Cycle," Trans. Am. Nucl. Soc., Vol. 92, p. 651, 2005.

[5] S. Yun and N. Z. Cho, "A Monte Carlo Depletion Method with Leakage Corrected Critical Spectrum," Trans. Korean Nucl. Soc, Spring Meeting, Jeju, Korea, May 21-22, 2009.
[6] N. Z. Cho and J. Chang, "Some Outstanding Problems in Neutron Transport Computation," Nucl. Eng. Tech., Vol. 41, p. 381, 2009. http://article.nuclear.or.kr/pub/jknspaper. php?jid=JK0410381

[ 7 ] S. Yun and N. Z. Cho, "Study of Monte Carlo Depletion under Leakage-Corrected Critical Spectrum," Trans. Am. Nucl. Soc., Vol. 101, p. 703, 2009.

[ 8 ] X-5 Monte Carlo Team, "MCNP A General Monte Carlo N-Particle Transport Code, Version 5," Los Alamos National Laboratory, 2003.

[9 ] E. E. Lewis and W. F. Miller, Jr., Computational Methods of Neutron Transport, John Wiley \& Sons, New York, 1984.

[10] N. Z. Cho, S. Yun and J. Lee, "Generation of Homogenized Nodal Parameters by Monte Carlo Method with Non-Zero Leakage Spectra in Local/Global Iteration Framework," Trans. Am. Nucl. Soc., Vol. 101, p. 707, 2009.

[11] "Benchmark Problems in Reactor and Particle Transport Physics," http://nurapt.kaist.ac.kr/lab/kr/Eng/top3.htm, 2000 . 